\title{
8.9 hr Rotation in the Partly Burnt Runaway Stellar Remnant LP 40-365 (GD 492)
}

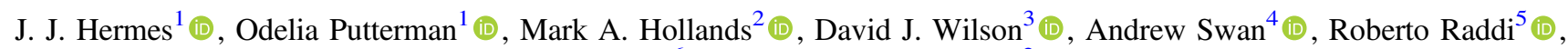 \\ Ken J. Shen ${ }^{6}$ (D), and Boris T. Gänsicke ${ }^{2}$ (D) \\ ${ }^{1}$ Department of Astronomy \& Institute for Astrophysical Research, Boston University, 725 Commonwealth Avenue, Boston, MA 02215, USA; jjhermes@bu.edu \\ ${ }^{2}$ Department of Physics, University of Warwick, Coventry CV4 7AL, UK \\ ${ }^{3}$ McDonald Observatory, University of Texas at Austin, Austin, TX 78712, USA \\ ${ }^{4}$ Department of Physics \& Astronomy, University College London, Gower Street, London WC1E 6BT, UK \\ ${ }^{5}$ Departament de Física, Universitat Politècnica de Catalunya, c/Esteve Terrades 5, E-08860 Castelldefels, Spain \\ ${ }^{6}$ Department of Astronomy and Theoretical Astrophysics Center, University of California, Berkeley, CA 94720, USA \\ Received 2021 April 14; revised 2021 May 11; accepted 2021 May 13; published 2021 June 7
}

\begin{abstract}
We report the detection of $8.914 \mathrm{hr}$ variability in both optical and ultraviolet light curves of LP 40-365 (also known as GD 492), the prototype for a class of partly burnt runaway stars that have been ejected from a binary due to a thermonuclear supernova event. We first detected this $1.0 \%$ amplitude variation in optical photometry collected by the Transiting Exoplanet Survey Satellite (TESS). Reanalysis of observations from the Hubble Space Telescope at the TESS period and ephemeris reveal a 5.8\% variation in the ultraviolet of this $9800 \mathrm{~K}$ stellar remnant. We propose that this $8.914 \mathrm{hr}$ photometric variation reveals the current surface rotation rate of LP 40-365, and is caused by some kind of surface inhomogeneity rotating in and out of view, though a lack of observed Zeeman splitting puts an upper limit on the magnetic field of $<20 \mathrm{kG}$. We explore ways in which the present rotation period can constrain progenitor scenarios if angular momentum was mostly conserved, which suggests that the survivor LP 40-365 was not the donor star but was most likely the bound remnant of a mostly disrupted white dwarf that underwent advanced burning from an underluminous (Type Iax) supernova.
\end{abstract}

Unified Astronomy Thesaurus concepts: Supernova remnants (1667); White dwarf stars (1799); Stellar rotation (1629)

\section{Introduction}

LP 40-365 (also known as GD 492) is an exceptionally peculiar star: it is one of the most metal-rich stars known, with an atmosphere dominated by oxygen and neon that is also abundant in heavy elements from partial oxygen and silicon burning, with no detectable hydrogen or helium (Vennes et al. 2017). Most peculiarly, it is rapidly departing the Galaxy, with a rest-frame velocity of $v_{\mathrm{rf}} \simeq 852 \mathrm{~km} \mathrm{~s}^{-1}$ that makes it unbound to the Milky Way; unlike hypervelocity stars (Brown 2015), LP 40-365 almost certainly did not come from the Galactic center (Raddi et al. 2018a). Currently, it appears to be an isolated star without radial-velocity variability, to a limit of roughly $2-3 \mathrm{~km} \mathrm{~s}^{-1}$ (Raddi et al. 2018b).

LP 40-365, the prototype to a small but growing class of similar objects (Raddi et al. 2019), derives these peculiarities because it was likely involved in a thermonuclear supernova event that ejected it from a short-period binary system. The original discoverers suggested that stars like LP 40-365 are most likely the bound remnants of partially disruptive thermonuclear supernovae, underluminous explosions often referred to as Type Iax supernovae (Vennes et al. 2017). Type Iax supernovae are thought to arise from an asymmetrically ignited deflagration in a Chandrasekhar-mass white dwarf that does not fully disrupt the star and leaves behind a bound remnant (e.g., Jordan et al. 2012; Foley et al. 2013; Fink et al. 2014; Jha 2017).

Gaia has enabled discovery of another class of runaways, the so-called "dynamically driven double-degenerate double-detonation" $\left(D^{6}\right)$ stars, which are moving with such high velocities $\left(>1000 \mathrm{~km} \mathrm{~s}^{-1}\right)$ they must be the surviving donor stars in double white dwarf systems that underwent thermonuclear supernovae (Shen et al. 2018b). In contrast, it is hard to constrain with certainty whether the LP 40-365-like stars were the donor or accretor in a close binary, since detailed abundance analyses are complicated by highly uncertain metal diffusion times and nucleosynthetic yields. Most aspects of abundance analysis suggests that these objects are the bound remnants of the actual white dwarf in which the partial deflagration occurred (Raddi et al. 2018b). However, the LP 40-365-like stars have also been analyzed as the polluted, inflated remnants of hot subdwarf donors in short-period progenitor systems (Bauer et al. 2019).

One useful boundary condition that could help us constrain the progenitor system for LP 40-365 would be the final rotation rate of the stellar remnant. The highest-resolution spectroscopic observations of LP 40-365 have only put an upper limit on the rotation velocity $\left(v_{\mathrm{rot}} \sin i<50 \mathrm{~km} \mathrm{~s}^{-1}\right)$ that implies a rotation period longer than $4 \mathrm{hr}$, based on current radius estimates (Raddi et al. 2018b).

We report here the significant detection of photometric variability in LP 40-365 at a period of $8.914 \mathrm{hr}$, present in an optical light curve collected by the Transiting Exoplanet Survey Satellite (TESS), with additional confirmation from an ultraviolet light curve from archival observations collected by the Hubble Space Telescope (HST) and possible detection in a near-infrared light curve from data collected by the Wide-field Infrared Survey Explorer (WISE).

We interpret this $8.914 \mathrm{hr}$ signal to correspond to the current surface rotation rate of LP 40-365. Section 2 and Section 3 of this Letter detail our observations and analysis, respectively. We conclude with a discussion of how the final rotation period we observe now in LP 40-365 can inform our understanding of the progenitor conditions that led to the formation of this partly burnt runaway stellar remnant. 

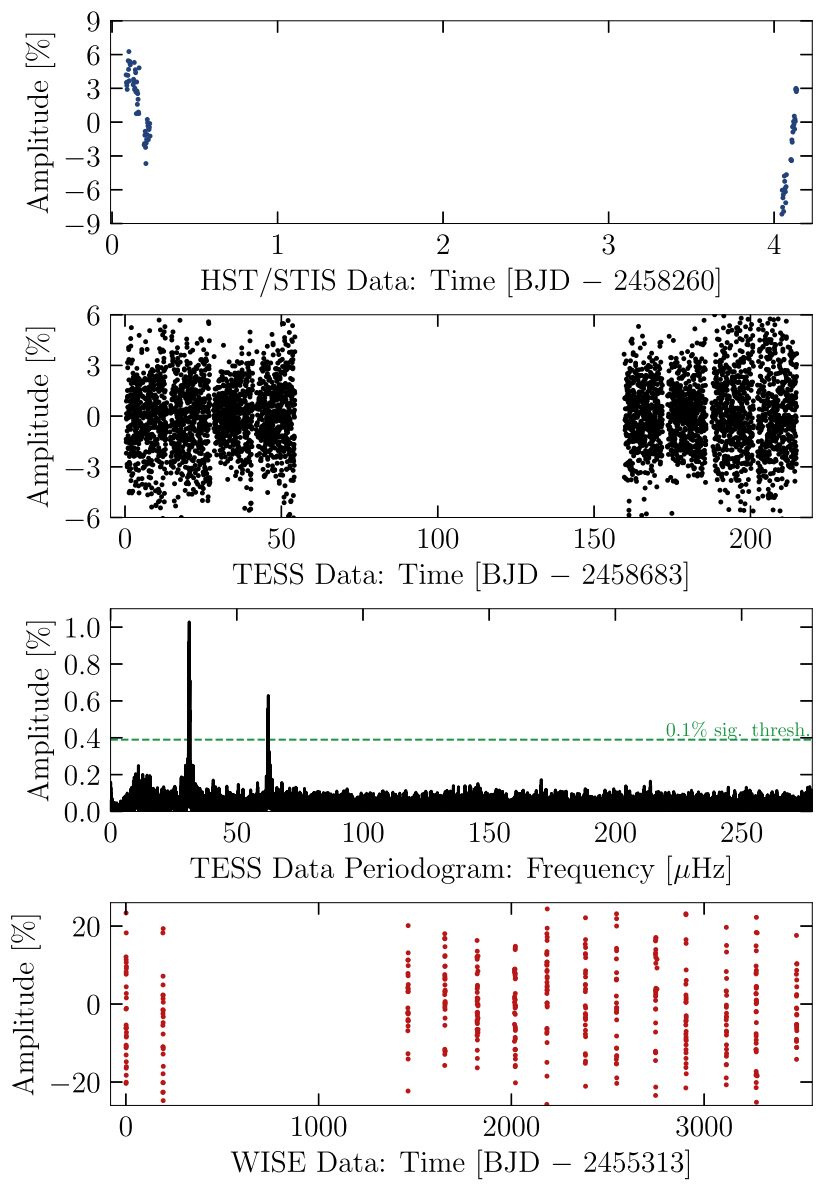

Figure 1. Full light curves of LP 40-365 used in this analysis. Top panel: five orbits of HST/STIS ultraviolet observations from 2018 May. Second panel: four sectors of TESS full-frame images from 2019 July to 2020 February. Third panel: periodogram of TESS observations revealing a dominant period at $8.914 \mathrm{hr}$ and its second harmonic. Bottom panel: more than $9.5 \mathrm{yr}$ of WISE infrared photometry from 2010 April to 2019 November.

\section{Observations and Reductions}

\subsection{TESS Observations}

LP 40-365 (TIC 198510602, $T=15.5 \mathrm{mag}$ ) was observed by TESS for nearly four months, in Sector 14 (2019 July 18 to 2019 August 14), Sector 15 (2019 August 15 to 2019 September 10), Sector 20 (2019 December 24 to 2020 January 20), and Sector 21 (2020 January 21 to 2020 February 18), all at 30 minute cadences in the full-frame images (it was not targeted by any shorter, 2 minute cadence observations). The light curves for all four sectors were extracted using the python package eleanor (Feinstein et al. 2019), and analyzed using the lightkurve package (Lightkurve Collaboration et al. 2018). TESS utilizes a broad, red optical filter centered at roughly $7865 \AA$.

The second panel of Figure 1 shows the full TESS light curve. Gaps in the data are present roughly every 13.7 days during each regular data download per orbit, and increased scatter due to reflected light is often present before the data downloads when the spacecraft is closer to Earth.

Our light curves were extracted using a fixed aperture, tuned for each sector depending on the spacecraft roll angle. We used a 3 px aperture for Sector 14, a 5 px aperture for Sectors 15 and 20, and a 2 px aperture for Sector 21. This changes the amount of extra light from background sources in the large pixel size
(>21 $\operatorname{arcsec}$ pixel $^{-1}$ ) for each sector, which makes comparing the relative amplitude from each sector more difficult. Therefore, the relative flux in our full light curve in Figure 1 was normalized to the median flux for each sector. No amplitude corrections have been made for possible crowding in the large sky area covered by these apertures, which may result in a suppressed amplitude in the TESS bandpass caused by flux dilution. Our full TESS light curve has 4338 points over 214.4 days, for a roughly $42 \%$ duty cycle.

\subsection{HST Observations}

We targeted LP 40-365 with the Space Telescope Imagining Spectrograph (STIS) instrument on board HST over five orbits in 2018 May, which were first described and analyzed in Raddi et al. (2019). The observations were proposed to analyze the ultraviolet spectrum and better constrain the effective temperature of LP 40-365, as well as improve our elemental abundance analysis.

We have reanalyzed these $15,253 \mathrm{~s}$ of STIS observations taken with the G230L grating by taking advantage of their time-tagged photon collection (Woodgate et al. 1998). We interacted with these data using the python package stistools ${ }^{7}$ available from the Space Telescope Science Institute. We binned the data into 3 minute intervals, then summed the spectra of each in the wavelength range with the most signal to noise $(\mathrm{S} / \mathrm{N}), 1700-3100 \AA$. This effectively created an ultraviolet light curve with 3 minute exposures and an effective central wavelength of $2520 \AA$. We corrected the light curve to barycentric time using Astropy (Astropy Collaboration et al. 2013). The full HST/STIS light curve has 82 points over 4.05 days, for a roughly $4 \%$ duty cycle, and is shown in the top panel of Figure 1.

HST is in constant orbit around the Earth, with natural changes in the focus of instruments like STIS due to thermal evolution (often described as breathing; e.g., Sing et al. 2013). Since our TESS observations revealed variability at much longer timescales than the HST orbit we have not corrected for this effect. However, it likely has added increased scatter on timescales shorter than 95.4 minutes.

\subsection{WISE Observations}

We constructed an infrared light curve for LP 40-365 using data from the WISE spacecraft, which is performing an all-sky survey, visiting most sources twice per year (Wright et al. 2010). We queried the AllWISE Multiepoch Photometry Table and NEOWISE-R Single Exposure Source Table, ${ }^{8}$ obtaining photometry in the $W 1$ and $W 2$ bands, taken during the decade 2010-2019. Here we only analyzed the higher-S/N observations in the $W l$ band, which has an effective wavelength of roughly $3.4 \mu \mathrm{m}$.

The WISE scanning pattern resulted in on average 32 separate $7.7 \mathrm{~s}$ exposures per visit for LP 40-365. Visits are spaced about six months apart, and last between two and eight days. Photometry is often analyzed after combining exposures within each visit (e.g., Swan et al. 2019; Secrest \& Satyapal 2020), but we kept our observations unbinned, resulting in the relatively large scatter seen in the bottom panel of Figure 1. Still, each

\footnotetext{
https://stistools.readthedocs.io

8 Available at https://irsa.ipac.caltech.edu/frontpage/.
} 
exposure used has a formal $\mathrm{S} / \mathrm{N}>4$, with an average $\mathrm{S} / \mathrm{N}$ of 9. We corrected the light curve to barycentric time using Astropy.

The point-spread function in the $W l$ band has an FWHM of 6.1 arcsec, and thus source confusion is a potential concern (Dennihy et al. 2020). However, no excess infrared flux is observed (Raddi et al. 2018b), and the median discrepancy between measured positions and those predicted from Gaia astrometry is 0.3 arcsec. The full WISE/W1 light curve has 451 points over 3480 days, for a roughly $0.001 \%$ duty cycle, and is shown in the bottom panel of Figure 1.

\section{Light-curve Analysis}

A Lomb-Scargle periodogram of all four sectors of TESS data, shown in the third panel of Figure 1, reveals two significant peaks: one dominant at roughly $8.90 \mathrm{hr}$ and another peak at exactly half that period, roughly $4.45 \mathrm{hr}$. The $0.1 \%$ significance threshold shown, at $0.39 \%$ amplitude, was bootstrapped by keeping the time sampling identical but randomly shuffling the flux values in the light curve, noting the maximum peak for $99.9 \%$ of all light curves from noise alone, as described in Hermes et al. (2015).

A simultaneous nonlinear least-squares fit of all TESS data, forcing the second frequency to be exactly twice that of the dominant signal, finds a best-fit period of $8.91405 \pm 0.00035 \mathrm{hr}$. We have performed the same weighted least-squares fit to the combined TESS+WISE data set, which yields a more precise period, which we adopt throughout: $8.914122 \pm 0.000020 \mathrm{hr}$. From the first observed time of minimum in TESS we define the ephemeris as

$$
\mathrm{BJD}_{\mathrm{TDB}}=2458683.7076(23)+0.37142177(83) E .
$$

We fix this period and compute a linear least-squares fit for each of the three light curves to define the amplitude of variability in each data set. We find an ultraviolet amplitude of $5.79 \pm 0.21 \%$ from the HST data, an optical amplitude of $1.038 \pm 0.042 \%$ from the TESS data, and an infrared amplitude of $1.94 \pm 0.81 \%$ from the WISE data. The uncertainties on the amplitudes are purely formal, and do not include any systematic effects.

We use the ephemeris to phase all light curves to the same baseline, which we show visually in Figure 2. The best-fit amplitudes reported above are shown visually as a faint green dashed line in each panel. The phase-folded, binned WISE data are best fit with an amplitude larger than the full unbinned light curve $(2.20 \pm 0.87 \%)$.

The significant detection of the second harmonic of the $8.914 \mathrm{hr}$ fundamental period in the periodogram in Figure 1 suggests an asymmetry in the light curve. Nonsinusoidal light curves have been observed in other white dwarfs exhibiting photometric variability from surface spots (e.g., Hermes et al. 2017a, 2017b; Hoard et al. 2018), and the second harmonic may be modeled in the future to constrain the inclination to the star. The folded light curves in Figure 2 show that the photometric variations are mostly sinusoidal; excluding this harmonic does not change our reported ephemeris or measured amplitudes within the stated uncertainties.

We detect $8.914 \mathrm{hr}$ variability with significance in multiple data sets; the variability appears phase coherent over multiple years, as well as between the optical and ultraviolet wavelengths. In 2017 we observed LP 40-365 for $4 \mathrm{hr}$ from the 2 meter Liverpool Telescope but saw no optical variability to a limit of roughly $0.3 \%$
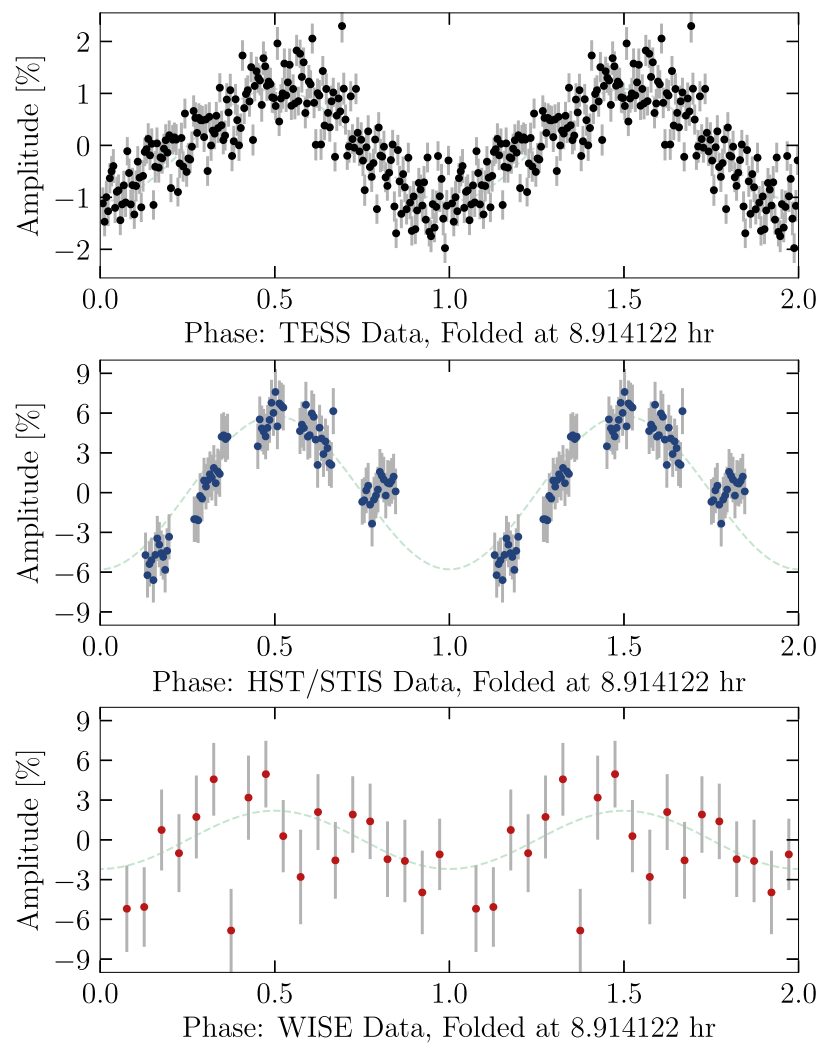

Figure 2. Folded light curves for LP 40-365, with each phased using the ephemeris defined from the WISE+TESS observations and repeated for clarity. Top panel: all TESS data, which have an effective wavelength of roughly $7900 \AA$ A, binned into 200 phase bins. Middle panel: all HST/STIS data, unbinned, which have an effective wavelength of roughly $2520 \AA$. Bottom panel: all WISE/WI data binned into 20 phase bins, which have an effective wavelength of roughly $3.4 \mu \mathrm{m}$.

amplitude (Raddi et al. 2018b); it is likely the signal we see here was too long to detect from that light curve taken through a $V+R$ band filter. We cannot certify that the variability observed in the WISE observations is formally significant, although it is suggestive that the phased data in Figure 2 shares the same photometric minimum as the TESS observations. Only $1 \%$ of field sources vary at the wavelengths covered by WI (Kozłowski et al. 2010; Polimera et al. 2018), so it is likely that the periodic variation we detect in WISE is from LP 40-365.

We reserve a more detailed analysis of the amplitude ratios between the ultraviolet-optical-infrared data sets until we can properly assess crowding in the TESS observations, which could contribute to flux dilution and thus optical amplitude suppression. It is reasonable that the ultraviolet variations have higher amplitude than the optical variations, given that this is a $9800 \mathrm{~K}$ stellar remnant (Raddi et al. 2019).

\section{Discussion and Conclusion}

We interpret the coherent $8.914 \mathrm{hr}$ photometric variability in LP 40-365 as the surface rotation period of this runaway stellar remnant. Spots caused by surface inhomogeneities on white dwarfs have been seen in a growing number of stars observed with space-based photometry from Kepler and TESS (Hermes et al. 2017a), especially in the presence of strong magnetic fields (Brinkworth et al. 2013). Without knowing the topology of the magnetic field of this white dwarf, the inferred 
rotation period could be off by a factor of 2; our inference of a $8.914 \mathrm{hr}$ rotation rate assumes a dipole field geometry.

We have reanalyzed the highest-resolution optical spectra collected from the R1200R grating from the 4.2 meter William Herschel Telescope on La Palma, described in Raddi et al. (2018b). The sharp metal lines observed in that spectrum allowed us to put an upper limit on rotation of $v_{\text {rot }} \sin i<50 \mathrm{~km} \mathrm{~s}^{-1}$ (we would infer a rotation velocity of roughly $22 \mathrm{~km} \mathrm{~s}^{-1}$ from an $8.9 \mathrm{hr}$ rotation period). Similarly, using the observed shape of the calcium triplet between $8498 \AA$ and $8662 \AA$ (which is particularly sensitive to small magnetic fields), we do not detect any lineprofile changes caused by Zeeman splitting from a magnetic field. This allows us to put an upper limit on the magnetic field in LP 40 -365 of $<20 \mathrm{kG}$. Such a small field may not preclude formation of spots from surface inhomogeneities-for example, we observe a spot from rotation in a pulsating white dwarf which has a global magnetic field $<10 \mathrm{kG}$ (Hermes et al. 2017b).

The best estimates of the parameters of LP 40-365 are detailed in Raddi et al. (2019): $T_{\text {eff }}=9800 \pm 300 \mathrm{~K}, \log g=5.5 \pm$ 0.3 [cgs], $L=0.20 \pm 0.04 L_{\odot}, R=0.16 \pm 0.01 R_{\odot}$, and $M=$ $0.28_{-0.14}^{+0.28} M_{\odot}$. Using these atmospheric parameters, we find the gas pressure at an optical depth of $\tau=2 / 3$ is $10^{4.7}$ dyne $\mathrm{cm}^{-2}$. The magnetic pressure will exceed this gas pressure at roughly $1 \mathrm{kG}$, so our $20 \mathrm{kG}$ upper limit allows for a field that can measurably influence the atmospheric structure at the photosphere.

It is likely that the partly burnt remnant LP 40-365 is still highly inflated; tracing back the kinematics yields an expected flight time of this star away from the Galactic disk of 5.3 Myr, which is considerably shorter than the Kelvin-Helmholtz timescale of $32 \mathrm{Myr}$. Considering this radius inflation, we have explored how the current rotation period can constrain progenitor scenarios if angular momentum in the system is mostly conserved.

We first consider if LP 40-365 was the white dwarf that underwent a thermonuclear event; in this first case we assume the progenitor star had a mass near the Chandrasekhar limit $\left(M_{i}>1.3 M_{\odot}\right)$, with a corresponding radius of $R_{i}<0.004 R_{\odot}$ (Althaus et al. 2005). In this scenario, most of the mass from this star was lost in the thermonuclear event, and the radius has increased both because the lower-mass white dwarf is less degenerate but also because of eventual entropy release as the remnant evolves (e.g., Zhang et al. 2019). Assuming angular momentum transport is efficient and mostly conserved implies an initial rotation period of less than 2 minutes. This is very fast compared to typical isolated white dwarfs, which rotate with periods from 0.5-2 days (Hermes et al. 2017c), but is still much slower than breakup velocity (which would have a spin period of order seconds).

A 2 minute initial spin period is likely faster than the expected orbital period at explosion: LP 40-365 likely had an ejection velocity of roughly $v_{\mathrm{ej}} \simeq 600 \mathrm{~km} \mathrm{~s}^{-1}$, which suggests a progenitor orbital period of roughly 10-30 minutes for most configurations (Raddi et al. 2018a). However, such spin-up would not be surprising for an accretor: spin-up is commonly expected (e.g., Ritter 1985) and observed (e.g., Lopes de Oliveira et al. 2020) in cataclysmic variables with high masstransfer rates onto accreting white dwarfs.

We also consider if LP 40-365 was the donor star ejected from a once-compact binary system; this scenario was modeled in detail by Bauer et al. (2019). They explored two different progenitors at different orbital periods: their two hot subdwarf donor models (a $0.344 M_{\odot}$ donor onto a $0.927 M_{\odot}$ accretor and a $0.233 M_{\odot}$ donor onto a $0.779 M_{\odot}$ accretor) predict rapid rotation for the donor remnant $10 \mathrm{Myr}$ after explosion; all donor remnants with final masses $>0.2 M_{\odot}$ end up with rotation periods faster than roughly $1 \mathrm{hr}$, much faster than $8.9 \mathrm{hr}$. (Shocks and interactions with high ejecta kinetic energies will strip some or even most of the donor mass by the time it becomes a remnant, and the donor models specified above are predetonation masses.)

Combining the Keplerian orbital velocity of the donor with the expectations that it is Roche-lobe filling allowed Bauer et al. (2019) to predict the radius of the donor at explosion in terms of its orbital velocity. The expected range of orbital velocities for LP 40-365 range from 600-800 $\mathrm{km} \mathrm{s}^{-1}$ (Raddi et al. 2019), which would predict a donor radius of $0.055-0.16 R_{\odot}$ in orbital periods ranging from roughly 5-20 minutes.

The expected radius of the donor computed via the Rochelobe radius depends on the unknown mass ratio of the system (Eggleton 1983) at explosion; ranges for $M_{1}$ are unlikely to exceed $0.2-0.5 M_{\odot}$ for a core-He-burning subdwarf donor, and $M_{2}$ are unlikely to exceed $0.85-1.4 M_{\odot}$ given that white dwarf detonations could be sub-Chandrasekhar in a subdwarf donor scenario (Shen et al. 2018a). All told, we cannot explain the high ejection velocity with the comparatively slow rotation period currently observed in LP 40-365 if this is the donor remnant that was tidally locked in a short-period binary; the progenitor requires significantly more radius inflation to slow its rotation to the period we observe today.

There are two assumptions that could affect our inferences. It is likely a subdwarf donor would be close to tidally locked in these short-period binaries (Preece et al. 2018), but breaking this assumption would affect initial spin-period estimates. Moreover, we cannot exclude a scenario in which the donor was highly inflated $\left(R>20 R_{\odot}\right)$ after explosion and lost significant angular momentum due to mass loss and magnetic braking. This phase should be relatively short-lived $\left(<10^{5} \mathrm{yr}\right.$, e.g., Pan et al. 2013), but the mass loss may be significant enough to complicate the assumption of angular momentum conservation.

That said, if angular momentum is mostly conserved, we find it less likely that LP 40-365 represents the donor in a disrupted compact binary. Most likely, it is still experiencing a dramatic radius expansion best explained by it being the once-accreting white dwarf that itself underwent a thermonuclear event. This radius inflation is also supported by another much hotter LP 40 -365-like star, J1825-3757, which has a similar mass as LP 40-365 but a radius at least a factor of 3 larger (Raddi et al. 2019). The event that disrupted the binary system caused both components to sling-shot apart at high speed. That thermonuclear event also led to partial burning in the progenitor star to LP 40-365, as proposed by Vennes et al. (2017) and Raddi et al. (2018a, 2018b), based in part on the currently observed heavy-element abundances.

Clarifying the role of LP 40-365 in the explosion is important to further interpreting the abundances, especially to connect to expected nucleosynthetic yields (e.g., Leung \& Nomoto 2020). This connection is vital to help us better understand the peculiar properties of the class of partially burnt stellar remnants, the LP 40-365 stars (Raddi et al. 2019). 
We acknowledge helpful comments from the anonymous referee, and thank Evan Bauer for valuable discussions. Support for this work was in part provided by NASA TESS Cycle 2 grant 80NSSC20K0592. M.A.H. has received funding from the European Research Council under the European Union's Horizon 2020 research and innovation program n. 677706 (WD3D). A.S. has received support from Science and Technology Facilities Council grant ST/ R000476/1. R.R. has received funding from the postdoctoral fellowship program Beatriu de Pinós, funded by the Secretary of Universities and Research (Government of Catalonia) and by the Horizon 2020 program of research and innovation of the European Union under the Maria Skłodowska-Curie grant agreement No. 801370. K.J.S. was supported in part by NASA through the Astrophysics Theory Program (NNX17AG28G). B.T.G. was supported by the UK STFC grant ST/T000406/1 and by a Leverhulme Research Fellowship.

This Letter includes data collected by the TESS mission. Funding for the TESS mission is provided by the NASA's Science Mission Directorate. Based on observations made with the NASA/ESA Hubble Space Telescope, obtained from the Data Archive at the Space Telescope Science Institute, which is operated by the Association of Universities for Research in Astronomy, Inc., under NASA contract NAS 5-26555. These observations are associated with program \#15431, and funding in part provided by programs \#15871 and \#15918. This publication makes use of data products from the Wide-field Infrared Survey Explorer, which is a joint project of the University of California, Los Angeles, and the Jet Propulsion Laboratory/California Institute of Technology, funded by the National Aeronautics and Space Administration.

\section{ORCID iDs}

J. J. Hermes (DD https://orcid.org/0000-0001-5941-2286 Odelia Putterman (iD https://orcid.org/0000-0002-8935-0431

Mark A. Hollands (ib https://orcid.org/0000-0003-0089-2080

David J. Wilson (iD https://orcid.org/0000-0001-9667-9449

Andrew Swan (iD https://orcid.org/0000-0001-6515-9854

Roberto Raddi (1) https://orcid.org/0000-0002-9090-9191

Ken J. Shen (i) https://orcid.org/0000-0002-9632-6106

Boris T. Gänsicke (iD https://orcid.org/0000-0002-2761-3005

\section{References}

Althaus, L. G., García-Berro, E., Isern, J., \& Córsico, A. H. 2005, A\&A, 441, 689

Astropy Collaboration, Robitaille, T. P., Tollerud, E. J., et al. 2013, A\&A, 558, A33

Bauer, E. B., White, C. J., \& Bildsten, L. 2019, ApJ, 887, 68

Brinkworth, C. S., Burleigh, M. R., Lawrie, K., Marsh, T. R., \& Knigge, C. 2013, ApJ, 773, 47

Brown, W. R. 2015, ARA\&A, 53, 15

Cardoso, J. V. d. M., Hedges, C., et al. 2018, Lightkurve: Kepler and TESS Time Series Analysis in Python, Astrophysics Source Code Library, record, ascl:1812.013

Dennihy, E., Farihi, J., Fusillo, N. P. G., \& Debes, J. H. 2020, ApJ, 891, 97 Eggleton, P. P. 1983, ApJ, 268, 368

Feinstein, A. D., Montet, B. T., Foreman-Mackey, D., et al. 2019, PASP, 131, 094502

Fink, M., Kromer, M., Seitenzahl, I. R., et al. 2014, MNRAS, 438, 1762

Foley, R. J., Challis, P. J., Chornock, R., et al. 2013, ApJ, 767, 57

Hermes, J. J., Gänsicke, B. T., Bischoff-Kim, A., et al. 2015, MNRAS, 451, 1701

Hermes, J. J., Gänsicke, B. T., Gentile Fusillo, N. P., et al. 2017a, MNRAS, 468, 1946

Hermes, J. J., Gänsicke, B. T., Kawaler, S. D., et al. 2017c, ApJS, 232, 23

Hermes, J. J., Kawaler, S. D., Bischoff-Kim, A., et al. 2017b, ApJ, 835, 277

Hoard, D. W., Howell, S. B., Roettenbacher, R. M., et al. 2018, AJ, 156, 119

Jha, S. W. 2017, in Handbook of Supernovae, ed. A. W. Alsabti \& P Murdin (Berlin: Springer), 375

Jordan, G. C. I., Perets, H. B., Fisher, R. T., \& van Rossum, D. R. 2012, ApJL, 761, L23

Kozłowski, S., Kochanek, C. S., Stern, D., et al. 2010, ApJ, 716, 530

Leung, S.-C., \& Nomoto, K. 2020, ApJ, 900, 54

Lopes de Oliveira, R., Bruch, A., Rodrigues, C. V., Oliveira, A. S., \& Mukai, K. 2020, ApJL, 898, L40

Pan, K.-C., Ricker, P. M., \& Taam, R. E. 2013, ApJ, 773, 49

Polimera, M., Sarajedini, V., Ashby, M. L. N., Willner, S. P., \& Fazio, G. G. 2018, MNRAS, 476, 1111

Preece, H. P., Tout, C. A., \& Jeffery, C. S. 2018, MNRAS, 481, 715

Raddi, R., Hollands, M. A., Gänsicke, B. T., et al. 2018a, MNRAS, 479, L96

Raddi, R., Hollands, M. A., Koester, D., et al. 2018b, ApJ, 858, 3

Raddi, R., Hollands, M. A., Koester, D., et al. 2019, MNRAS, 489, 1489

Ritter, H. 1985, A\&A, 148, 207

Secrest, N. J., \& Satyapal, S. 2020, ApJ, 900, 56

Shen, K. J., Boubert, D., Gänsicke, B. T., et al. 2018b, ApJ, 865, 15

Shen, K. J., Kasen, D., Miles, B. J., \& Townsley, D. M. 2018a, ApJ, 854, 52

Sing, D. K., Lecavelier des Etangs, A., Fortney, J. J., et al. 2013, MNRAS, 436, 2956

Swan, A., Farihi, J., \& Wilson, T. G. 2019, MNRAS, 484, L109

Vennes, S., Nemeth, P., Kawka, A., et al. 2017, Sci, 357, 680

Woodgate, B. E., Kimble, R. A., Bowers, C. W., et al. 1998, PASP, 110, 1183

Wright, E. L., Eisenhardt, P. R. M., Mainzer, A. K., et al. 2010, AJ, 140, 1868

Zhang, M., Fuller, J., Schwab, J., \& Foley, R. J. 2019, ApJ, 872, 29 